\title{
The redshift distribution of the X-ray background
}

\author{
A. M. Sołtan \\ Nicolaus Copernicus Astronomical Center, Bartycka 18, 00-716 Warsaw, Poland \\ e-mail: soltan@camk.edu.pl
}

Received 18 June 2008 / Accepted 4 September 2008

\begin{abstract}
Context. The X-ray background (XRB) is produced by a large number of faint sources distributed over a wide range of redshifts. The XRB carries information on the spatial distribution and evolution of these sources.

Aims. The goals are 1. to determine the redshift distribution of the soft X-ray background photons produced by all types of extragalactic sources, in order to relate fluctuations of the background to the large scale structures; 2 . to determine the redshift distribution of the soft XRB produced by AGN in order to calculate the evolution of the AGN X-ray luminosity density.

Methods. A set of major X-ray surveys was used to determine the redshift distributions of the X-ray sources selected at various flux levels. Simple analytic fits to the data allow us to determine the smooth relationship between the redshift distribution and the source flux. The redshift distribution of the integral XRB flux was obtained by averaging the fits over the source counts.

Results. It is shown that the distribution of extragalactic XRB photons in the $0.5-2 \mathrm{keV}$ band is adequately represented by the function: $\mathrm{d} n_{\mathrm{XRB}} / \mathrm{d} \log z=5.24 z^{1.52} \exp (-z / 0.63)$. The huge voids postulated to explain the cold spots in the CMB maps create dips in the total XRB flux. However, the expected magnitude of the effect is comparable to the fluctuation amplitude of the XRB generated by the individual sources contributing to the background. The cosmic evolution of the AGN X-ray luminosity density up to a redshift of $\sim 5$ is calculated in an elegant and straightforward way. Systematic uncertainties of the present method are assessed and shown to be small. At a redshift greater than one the present results could be compared directly with some recent estimates obtained in a standard way and the agreement between both methods is very good.
\end{abstract}

Key words. X-rays: diffuse background - X-rays: galaxies - galaxies: active

\section{Introduction}

The X-ray background (XRB) is generated mostly by discrete extragalactic sources (e.g. Lehmann et al. 2001; Kim et al. 2007, and references therein), predominantly by various types of active galactic nuclei (AGN) and clusters of galaxies. A question of flux, luminosity, and redshift distributions of these sources has been discussed in a great number of papers for the past 30 years. One of the major outcomes of these investigations is the conclusion that X-ray sources associated with the AGN are subject to strong cosmic evolution (e.g. Miyaji et al. 2000; Silverman et al. 2007, and references therein). As a result of the evolution, the redshift distribution of the XRB flux is wide. Thus, the integral $\mathrm{XRB}$ comprises the information on the large-scale distribution of the X-ray sources over a wide redshift range.

In the present paper the redshift distribution of the XRB photons is investigated in detail. The analysis is based on an extensive observational data selected from several published X-ray sky surveys. Convenient analytic approximations are applied to model the observed redshift histograms of the extragalactic $\mathrm{X}$-ray sources selected at several flux levels. These distributions are weighted by the source counts and summed up to obtain the redshift distributions of the integral XRB.

Next, the redshift distribution is used to define a relationship between the XRB signal and the large-scale fluctuations of the matter spatial distribution. The investigation has been raised by the recent report on the huge void generating a dip in the surface brightness of the radio background (Rudnick et al. 2007).
This void, responsible for the deficit of the radio surface brightness, also allegedly generates a cold spot in the CMB map via the late-time integrated Sachs-Wolfe effect. Although the careful statistical analysis by Smith \& Huterer (2008) has not confirmed the existence of this particular "cold spot" in the radio survey, a relationship between the large-scale features of the matter distribution and the integrated sky brightness in various energy bands is a problem deserving some attention.

Apart from the question of the XRB fluctuations induced by voids, the $\mathrm{XRB}$ redshift distribution is interesting per se, as it allows assessment of the evolution of the AGN phenomenon. A standard way to estimate the rate and type of this evolution is based on examining the X-ray luminosity functions determined at the consecutive redshift bins. Unfortunately, the X-ray surveys produce flux-limited rather than luminosity-limited samples of sources. In effect, luminosity functions at different redshifts cover different luminosity ranges. This in turn severely impedes estimates of the luminosity function over a wide range of luminosities and redshifts. The total level of nuclear activity in galaxies within unit volume is given by the integral of the X-ray luminosity function. The question of the AGN cosmic evolution constitutes one of the central problems of observational cosmology and has been investigated for the past forty years (this issue was for the first time recognized by Schmidt 1968). Here a question of the AGN evolution is addressed without the calculations of the X-ray luminosity function. The available observational data on X-ray source counts and redshifts are used to evaluate the redshift distribution as a function of source flux. This 
relationship and the source counts allow calculation of the redshift distribution of the total XRB and the integral luminosity density generated by the AGN as a function of redshift.

The organization of the paper is as follows. First, I present the formulae used in calculations. Next, in Sect. 3, the basic information on the observational material extracted from the various archives is given. Since the comprehensive characteristics of the data and the source catalogs are described in the original papers, only the basic properties of the material are presented here. The numerical fits to the observed distributions are obtained in Sect. 4. In that section the calculations of the redshift distribution of the XRB photons are described in detail. These results are applied in Sect. 5 to quantify the relationship between the voids and the XRB variations. In Sect. 6 the distribution of the XRB flux produced by AGN is used to calculate the evolution of the AGN activity. Finally, potential sources of errors inherent in the present method are discussed in Sect. 7.

The "canonical" standard cosmology is assumed throughout, with $H_{0}=70 \mathrm{~km} \mathrm{~s}^{-1} \mathrm{Mpc}^{-1}, \Omega_{\mathrm{m}}=0.3$, and $\Omega_{\Lambda}=0.70$.

\section{Basic relationships}

In the present approach the X-ray source catalogs are used to construct redshift distributions of the extragalactic sources as a function of the source flux. At this stage, a question of the source (absolute) luminosities is not addressed. Let $N(S)$ denotes the $\mathrm{X}$-ray source counts, i.e. number of sources brighter than $S$ in a unit solid angle, and $f_{S}(z)=\mathrm{d} n(z \mid S) / \mathrm{d} \log z$ is the redshift distribution of sources with flux $S$. Then, the redshift distribution of the XRB surface brightness, $b(z)$, is equal to:

$b(z)=\frac{1}{b} \int \mathrm{d} S f_{S}(z) S\left|\frac{\mathrm{d} N(S)}{\mathrm{d} S}\right|$,

where the integration covers the entire "interesting" range of source fluxes $S$ and $b$ denotes the integral background flux:

$b=\int b(z) \mathrm{d} \log z=\int \mathrm{d} S S\left|\frac{\mathrm{d} N(S)}{\mathrm{d} S}\right|$.

It is assumed that the $f_{S}(z)$ distributions are normalized:

$\int f_{S}(z) \mathrm{d} \log z=1$.

Here the integration limits cover the total range of redshifts occupied by X-ray sources. The actual limits of the "interesting" range of fluxes is discussed below.

The luminosity density, $\varepsilon(z)$, i.e. a total luminosity $L$ generated in a unit comoving volume, $V$ :

$\varepsilon(z)=\frac{\mathrm{d} L}{\mathrm{~d} V}$,

is related to the flux distribution $b(z)$ and the luminosity distance, $D_{L}(z)$ :

$\varepsilon(z) \frac{\mathrm{d} V}{\mathrm{~d} \log z}=4 \pi D_{L}^{2}(z) b(z)$

In this, and all the others relevant formulae, it is implicitly assumed that the average energy spectral index of sources generating the soft XRB $\gamma=-1$. In this case no $K$-correction is needed. Alternatively, if $\gamma \neq-1$, the luminosity density $\varepsilon(z)$ is defined in the observed $0.5-2.0 \mathrm{keV}$ band, what in the object's rest frame corresponds to the band shifted to higher energies by a factor
Table 1. The X-ray surveys selected for the analysis.

\begin{tabular}{lcccc}
\hline \hline Name & \multicolumn{1}{c}{ Flux limits } & \multicolumn{3}{c}{ Number of sources } \\
& $\left(\mathrm{erg} \mathrm{cm}^{-2} \mathrm{~s}^{-1}\right)$ & all & extragalactic & AGN \\
\hline RBS & $1.0 \times 10^{-12}-5.0 \times 10^{-11}$ & 1764 & 1054 & 681 \\
NEP & $5.0 \times 10^{-14}-1.0 \times 10^{-12}$ & 361 & 248 & 192 \\
RIXOS & $2.5 \times 10^{-14}-5.0 \times 10^{-13}$ & 393 & 318 & 235 \\
XMS & $1.0 \times 10^{-14}-2.0 \times 10^{-13}$ & 275 & 256 & 231 \\
CDFS & $5.0 \times 10^{-17}-1.0 \times 10^{-15}$ & 205 & 201 & 197 \\
CDFN & $1.5 \times 10^{-17}-5.0 \times 10^{-15}$ & 425 & 412 & 268 \\
\hline
\end{tabular}

of $(1+z)$. The rationale for such an attitude is discussed in detail by Miyaji et al. (2000).

The cosmological relationships between the comoving volume in a unit solid angle and the luminosity distance in a flat space with $\Lambda \neq 0$ is given by Hogg (1999):

$\frac{\mathrm{d} V}{\mathrm{~d} z}=\frac{c}{H_{0}} \frac{D_{L}^{2}}{(1+z)^{2} \sqrt{\Omega_{\mathrm{m}}(1+z)^{3}+\Omega_{\Lambda}}}$.

Combining Eqs. (5) and (6) we finally get:

$\varepsilon(z)=4 \pi \frac{H_{0}}{c} \frac{(1+z)^{2} \sqrt{\Omega_{\mathrm{m}}(1+z)^{3}+\Omega_{\Lambda}}}{\ln 10 z} b(z)$.

Thus, to calculate the distributions $b(z)$ and $\varepsilon(z)$, the source counts $N(S)$ and the functions $n(z \mid S)$ have to be determined using the observational material. In the next section the available $\mathrm{X}$-ray surveys are examined from this point of view.

\section{Observational material}

Because the high imaging efficiency of X-ray telescopes in the soft band and numerous extensive identifications programs, we concentrate on the X-ray band of $0.5-2.0 \mathrm{keV}$. At these energies a fraction of the background resolved into discrete sources exceeds $90 \%$ and is higher than in the other bands (e.g. Moretti et al. 2003; Brandt \& Hasinger 2005). Also a fraction of identified objects with measured spectroscopic or photometric redshifts is relatively high.

Equation (1) shows that the $b(z)$ distribution is sensitive to sources which perceptibly contribute to the XRB. Consequently, one needs to calculate the $n(z \mid S)$ functions over a quite wide range of fluxes. To achieve this objective I have examined numerous X-ray surveys and selected several major source catalogs for further analysis. The overall characteristics of those catalogs are listed in Table 1. A common name of the survey/catalog is given in Col. 1. From the each catalog, sources for further processing have been extracted within fixed range of fluxes defined on Col. 2. The numbers of all sources, extragalactic and AGN with known redshifts are given in Cols. 3-5, respectively.

Statistical requirements which have to be satisfied by the source samples to properly determine the $n(z \mid S)$ distribution are different than those for the luminosity function calculations. The individual sample should contain sources from possible narrow range of fluxes, but the sample has not to be flux limited. The sample provides unbiased estimate of the redshift distribution as long as the process of identification and redshift measurements does not introduce spurious correlation between flux and redshift.

\subsection{The ROSAT Bright Survey (RBS)}

The identification program of the brightest sources detected in the ROSAT All-Sky Survey, known as ROSAT Bright Survey, 


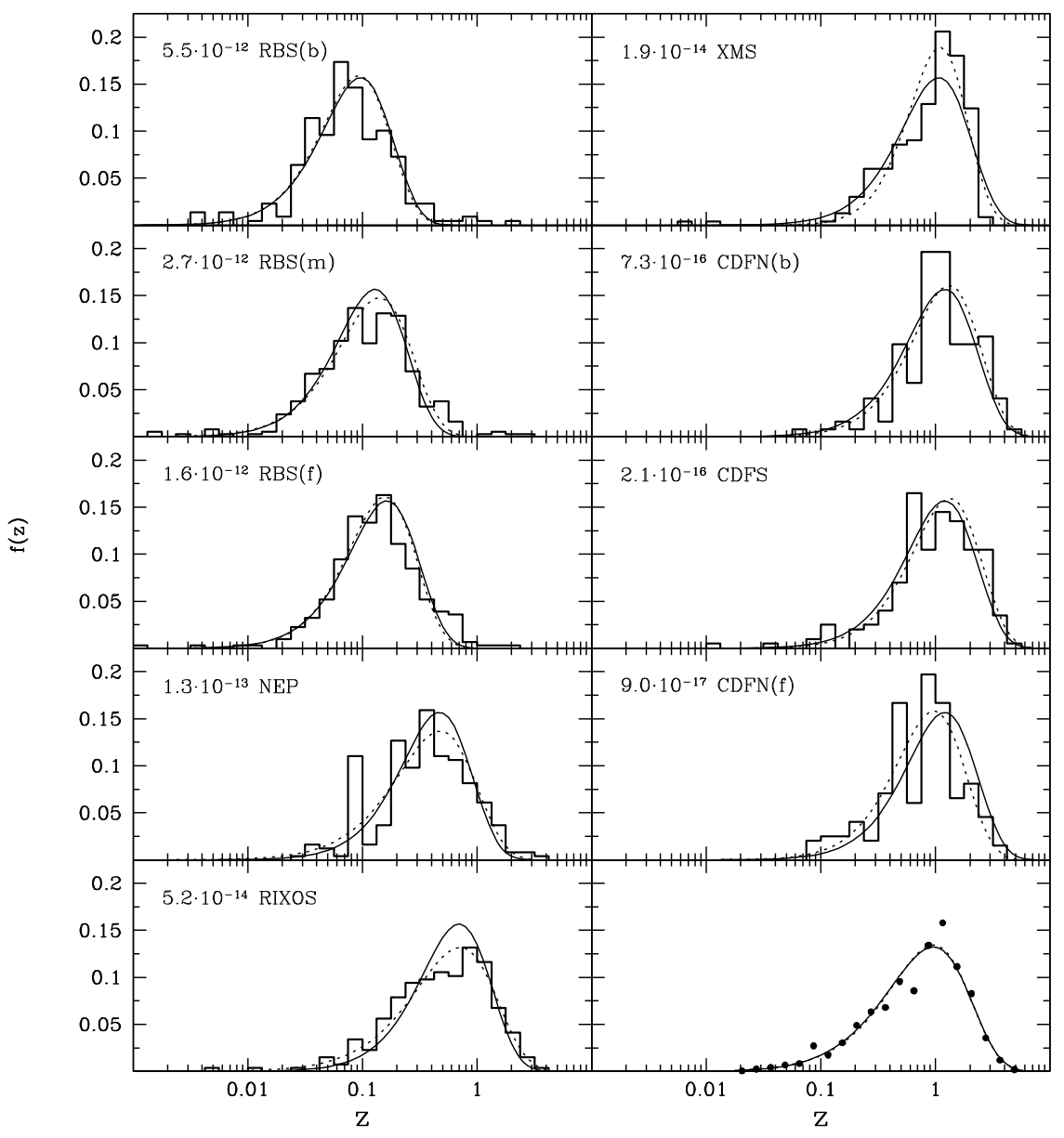

Fig. 1. Histograms with labels - the redshift distributions of extragalactic sources in 9 samples constructed in the present investigation. The data are binned with $\Delta \log z=0.125$; the integrals of all the histograms are normalized to unity. The sample designations and the median fluxes in cgs are given in the upper left corners; dotted curves best 3 parameter fits for the each sample separately; solid curves - the best model for all the samples. The lower right panel: solid curve - the model redshift distribution for the whole XRB (the normalization of the distribution is the same as for the histograms); dotted curve - analytic approximation of the model distribution; points - the distribution obtained by the linear interpolation between the raw histograms (see text for details). resulted in a sample of 2072 sources with the total count rate above $0.2 \mathrm{~s}^{-1}$ (Schwope et al. 2000). More than $99.5 \%$ of sources in the final catalog is identified. The survey covers high galactic latitudes $(|b|>30 \mathrm{deg})$. After the removal of the Virgo clusters and Magellanic Clouds regions, the catalog contains 2012 sources. In the energy band of $0.5-2.0 \mathrm{keV}, 1773$ RBS sources generate flux between $1.0 \times 10^{-12} \mathrm{erg} \mathrm{cm}^{-2} \mathrm{~s}^{-1}$ (hereafter cgs) and $5.0 \times 10^{-11} \mathrm{cgs}$. As one might expect, only for a small fraction of the RBS sources the redshifts are undetermined and relatively large number of sources is associated with galactic sources, mostly late type stars and cataclysmic variables.

Since the RBS sample covers rather wide range of fluxes, it is useful for our purposes to divide it into several subsamples with narrow flux limits and to estimate the $n(z \mid S)$ distribution for the each set separately. We define the bright source sample, labeled RBS(b), which contains sources with $4.0 \times 10^{-12}<S<$ $1.0 \times 10^{-11} \mathrm{cgs}$. Of $365 \mathrm{RBS}$ sources in this flux range, 129 is identified with galactic objects and for the other 15 sources redshifts have not been measured. Eventually, the sample contains 221 extragalactic objects with known redshifts. Nearly half of the sample, viz. 97 sources are identified with clusters of galaxies and normal galaxies. The median flux ${ }^{1}$ in the extragalactic subsample $S_{\mathrm{m}}=5.5 \times 10^{-12} \mathrm{cgs}$.

The flux limits of $2.0 \times 10^{-12}<S<4.0 \times 10^{-12} \mathrm{cgs}$ have been adopted for the medium flux sample, $\operatorname{RBS}(\mathrm{m})$. Within

\footnotetext{
1 The samples extracted from the RBS span over narrow ranges of fluxes and there is no major difference between the mean and median values. In some other samples investigated in this paper the median flux is distinctly smaller than the average. In those cases, the median flux is adopted as the argument in the $n(z \mid S)$ functions.
}

this flux range the RBS comprises of 669 objects including 272 galactic stars. Among the 397 extragalactic sources, 233 are identified with AGN with known redshifts. The median flux in the RBS(m) extragalactic sample, $S_{\mathrm{m}}=2.7 \times 10^{-12} \mathrm{cgs}$. The faint source sample, RBS(f), contains sources with $1.0 \times 10^{-12}<$ $S<2.0 \times 10^{-12} \mathrm{cgs}$. Of $599 \mathrm{RBS}$ sources in this flux range 268 is identified with galactic objects and 24 has unknown redshifts. Thus, the RBS(f) sample contains 307 sources extragalactic objects with the median flux of $1.6 \times 10^{-12} \mathrm{cgs}$.

The redshift distributions of sources in the RBS(b), (m), and (f) samples are shown in three upper left panels in Fig. 1. The integrals of the histograms for all the samples in Fig. 1 are normalized to unity. The distributions are plotted using logarithmic redshift bins with $\Delta \log z=0.125$. Each histogram is labeled with the survey name and the median flux in cgs. Analytic fits will be discussed in the next section.

\subsection{The ROSAT North Ecliptic Pole Survey (NEP)}

The deepest exposure of the ROSAT All-Sky Survey (RASS) is centered at the north ecliptic pole (Voges et al. 1999). The RASS of this region has been used to construct statistically well defined sample of X-ray sources above a flux limit $\sim 2 \times 10^{-14} \mathrm{cgs}$ (Henry et al. 2006) which have been followed-up by the optical observations (Gioia et al. 2003). The identification rate in the final catalog of 443 sources is very high (99.6\%).

Within the flux limits of $5.0 \times 10^{-14}$ and $1.0 \times 10^{-12} \mathrm{cgs}$ the NEP survey provided 361 sources. After excluding 113 galactic stars, we are left with 248 extragalactic sources; for 3 sources the redshift is unknown. The redshift histogram of 245 sources 
(including 53 clusters) is shown in Fig. 1; the median flux in this sample is equal to $1.3 \times 10^{-13} \mathrm{cgs}$.

\subsection{The ROSAT International X-ray/Optical Survey (RIXOS)}

This ROSAT medium-sensitivity survey consists of sources found in 82 PSPC pointing observations at high galactic latitudes $(|b|>28 \mathrm{deg})$. A flux limit of $3 \times 10^{-14} \mathrm{cgs}$ was adopted in 64 fields and $8 \times 10^{-14} \mathrm{cgs}$ in the remaining 18 fields. The source selection procedures, optical identifications and the final catalog are given by Mason et al. (2000).

For the purpose of the present analysis, 393 sources with fluxes between $2.5 \times 10^{-14}$ and $5 \times 10^{-13}$ cgs have been selected. Within these flux limits 75 sources are associated with galactic stars. Of the remaining 318 sources, the redshifts of three objects are unknown, and 49 sources are still unidentified. The redshift distribution of 266 sources (including 33 clusters) is shown in Fig. 1. The median flux in this subsample $S_{\mathrm{m}}=5.2 \times 10^{-14} \mathrm{cgs}$.

\subsection{The XMM-Newton serendipitous survey (XMS)}

The XMM-Newton serendipitous survey (XMS) has been constructed in a similar way as the RIXOS. More than 300 sources have been isolated in 25 high galactic latitude $(|b|>22 \mathrm{deg})$ pointings covering $\sim 3 \mathrm{deg}^{2}$ of the sky (Barcons et al. 2007). In the $0.5-2.0 \mathrm{keV}$ band the sample is complete above $1.5 \times$ $10^{-14} \mathrm{cgs}$, and contains many weaker sources.

I have extracted from the original catalog 275 sources with fluxes between $1.0 \times 10^{-14}$ and $2.0 \times 10^{-13} \mathrm{cgs}$. The sample is completely identified; it contains 19 stars and 2 clusters of galaxies. For 23 objects the redshifts are unknown. The redshift distribution of 233 sources is shown in Fig. 1. The median flux in this subsample $S_{\mathrm{m}}=1.9 \times 10^{-14} \mathrm{cgs}$.

\subsection{The Chandra Deep Field-South (CDFS)}

The 1 Ms Chandra observations known as the Chandra Deep Field South are described by Giacconi et al. (2002). The catalog of sources detected in this field by two independent algorithms contains 304 objects, of which 275 have determined fluxes in the $0.5-2.0 \mathrm{keV}$ band. For further processing 205 sources with fluxes in the range $5.0 \times 10^{-17}-1.0 \times 10^{-15} \mathrm{cgs}$ have been selected. Four sources are identified with stars. The redshifts either spectroscopic (Szokoly et al. 2004; Ravikumar et al. 2007) or photometric (Zheng et al. 2004) are known for 200 sources; one source remains unidentified.

The redshift distribution is shown in Fig. 1. The median flux in this sample $S_{\mathrm{m}}=2.1 \times 10^{-16} \mathrm{cgs}$. The sample contains one galaxy group; two other sources apparently are not associated with the activity in the galactic nuclei (Lehmer et al. 2006). It is likely, however, that more objects in the CDFS survey should be classified as off-nuclear sources. The available data do not allow for unambiguous separation of AGN and off-nuclear sources at the low flux levels in the CDFS. This reservation holds also for the CDFN samples below.

\subsection{The Chandra Deep Field-North (CDFN)}

The ultra deep Chandra field, 2 Ms exposure, CDFN, resulted in a catalog of 503 sources detected over 0.12 sq. deg (Alexander et al. 2003). Optical follow-up observations by Barger et al. (2003) have rendered a large number of spectroscopic and photometric redshifts. Several more redshifts are taken from
Reddy et al. (2006), Donley et al. (2007) and Georgakakis et al. (2007).

In the present investigation, the CDFN catalog has been divided into two samples of bright (b) and faint (f) sources. The (b) sample contains 181 sources between $2.5 \times 10^{-16}$ and $5.0 \times$ $10^{-15} \mathrm{cgs}$; ten sources have been identified with the galactic stars; at least one has been categorized as "starburst" galaxy (Georgakakis et al. 2007), for 49 objects the redshifts have not been measured. The sample consists of 122 sources, mostly AGN. The median flux in this subsample $S_{\mathrm{m}}=7.3 \times 10^{-16} \mathrm{cgs}$.

The faint CDFN sample has been selected between $1.5 \times$ $10^{-17}$ and $2.5 \times 10^{-16} \mathrm{cgs}$. Among 244 sources satisfying these flux limits, three sources have been identified with stars, 51 with the starburst galaxies (Georgakakis et al. 2007) and for 43 objects the redshifts have not been measured. The final sample used in the calculations contains 198 sources with 147 confirmed AGN. The median flux in the sample $S_{\mathrm{m}}=9.0 \times$ $10^{-17}$ cgs. The redshift histograms for the (b) and (f) samples are shown in Fig. 1.

The number of objects unidentified or without redshift is quite large in some samples. Hence one could expect that the corresponding redshift histograms are not representative for the whole population of sources at given flux. Below this question is de facto worked out where we construct an analytic function which simultaneously fits all the histograms.

\section{Approximations and fits}

The redshift distribution of sources selected at fixed flux, $n(z \mid S)$, is an intricate function of a number of parameters, such as the luminosity function, the relationship between the luminosity and observable flux, and the relationship between the volume and redshift. The luminosity function itself depends on redshift and both the latter relationships depend on the cosmological model. However, the existing estimators of the $n(z \mid S)$ function represented by the nine histograms in Fig. 1 are strongly affected/degraded by the statistical noise. It implies that a simple analytic function with $2-3$ free parameters will provide a statistically satisfactory fit to the observed distributions.

It appears that the histograms in Fig. 1 are adequately reproduced by:

$f_{S}(z)=f_{0} z^{\alpha} \mathrm{e}^{-z / z_{c}}$

where $f_{0}=f_{0}(S), \alpha=\alpha(S)$, and $z_{\mathrm{c}}=z_{\mathrm{c}}(S)$ are three parameters fitted to the histograms $n\left(z \mid S_{i}\right), i=1, \ldots, 9$.

The least square method was applied. First, the function $f_{S}(z)$ was linearized and the initial values of fitted parameters were chosen. Then, through the iterative procedure values of the parameters were refined. The uncertainties of the observed number of sources in the consecutive redshift bins were defined as square roots of the number of objects (assuming the Poissonian statistics). In the effort to minimize effects of the small number noise, the uncertainty for each bin was calculated taking the average counts in this bin and two neighboring bins. This procedure reduced statistical fluctuations of weights in the normal equations (which yield the least square solution) to the acceptable level. For empty bins, the expected number of sources based on the previous iteration were applied. The largest reduced $\chi^{2}$ of 2.213 was found for the NEP sample. This value results from the excess of objects in the $0.075-0.100$ redshift bin. The supercluster at the redshift 0.087 in the NEP region reported by Mullis et al. (2001) is responsible for the entire effect. The reduced $\chi^{2}$ for the remaining samples varies between 0.539 


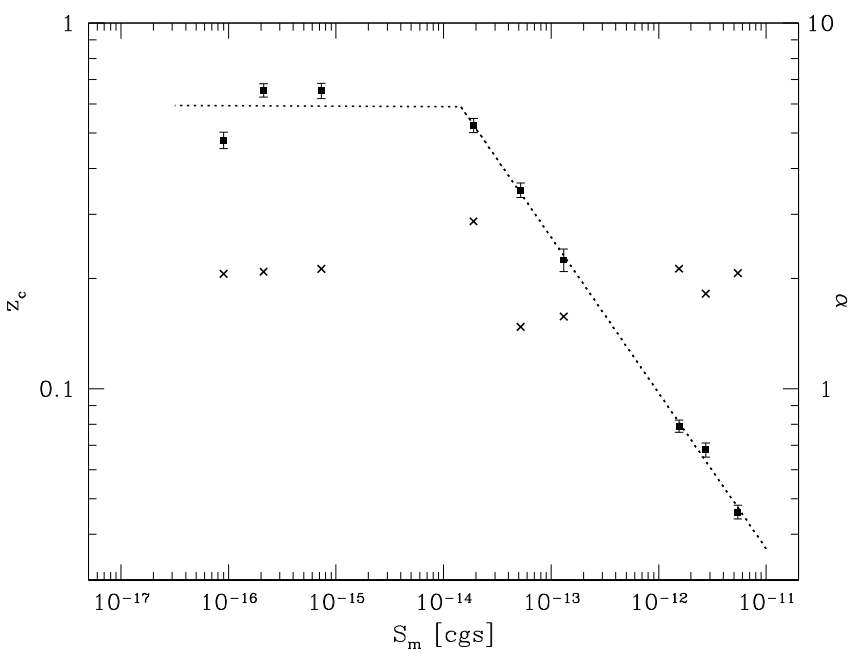

Fig. 2. Distributions of the best fitted parameters $\alpha$ and $z_{\mathrm{c}}$ vs. the median flux, $S_{\mathrm{m}}$. Points with error bars $-z_{\mathrm{c}}$ (left-hand ordinate), crosses $\alpha$ (right-hand ordinate). The assumed $z_{\mathrm{c}} \sim S$ relationships used to model the XRB redshift distribution is shown with the dotted lines (see text for details).

for RIXOS sample and 1.405 for RBS(m). In Fig. 1 fits for all the distributions are shown with the dotted curves. Apart from a few pronounced features visible in the plots which represent the large scale structures reported in the literature (e.g. Mullis et al. 2001; Barger et al. 2002; Gilli et al. 2003), analytic fits seem to adequately reproduce the observed distributions.

It is found that only $z_{\mathrm{c}}$ is strongly correlated with $S_{\mathrm{m}}$, while the fits do not indicate any statistically significant correlation between $\alpha$ and $S_{\mathrm{m}}$. In Fig. 2 the best fit values of $\alpha$ are shown with crosses. The labels and scale on the left-hand ordinate refer to $z_{\mathrm{c}}$, and on the right-hand - to $\alpha$. Since the simultaneous fitting of $\alpha$ and $z_{\mathrm{c}}$ introduces a spurious correlation between these two parameters, the $\alpha$ parameter has been fixed at the average value found for the 9 samples, $\bar{\alpha}=1.934$. Effectively, it means that the shape of the $n(z \mid S)$ function is fixed and the only dependence on $S$ is limited to the horizontal shift along the $z$ axis. In Fig. 2 the best fit parameters $z_{\mathrm{c}}$ found for the fixed $\alpha$ are plotted against the median flux $S_{\mathrm{m}}$. In agreement with the expectations, the $z_{\mathrm{c}}$ increases with diminishing flux $S_{\mathrm{m}}$ over a wide range of fluxes. However, a clear flattening of the relationship is observed below $\sim 10^{-14} \mathrm{cgs}$. This apparent absence of correlation between $z_{\mathrm{c}}$ and $S$ results from the well-known fact that in the X-ray surveys at low flux levels the maximum detected redshift remains stable while significantly increases fraction of intrinsically weak sources.

The points in the $z_{\mathrm{c}}-S_{\mathrm{m}}$ relationship above $\sim 10^{-14} \mathrm{cgs}$ seem to follow the power law. In the subsequent calculations it is assumed that this relationship is in fact well approximated by the power law in the whole range of fluxes between $10^{-14}$ and $10^{-11} \mathrm{cgs}$, although the data coverage is rather sparse. Using the least square method we get:

$\log z_{\mathrm{c}}=-(0.426 \pm 0.008) \log S_{\mathrm{m}}-(6.12 \pm 0.10)$.

Below $S \approx 10^{-14} \mathrm{cgs}$ the data are insufficient to delineate precisely the $z_{\mathrm{c}}-S_{\mathrm{m}}$ relationship. I have assumed tentatively that $z_{\mathrm{c}}$ remains constant and is equal to the average value found for three Chandra samples. The $z_{\mathrm{c}}$ in CDFN(b), CDFS and CDFN(f) are equal to $0.69,0.69$ and 0.48 , respectively. The average weighted by the uncertainties $\bar{z}_{\mathrm{c}}=0.59$. In Fig. 2 the model relationship $z_{\mathrm{c}}-S$ used in the calculations is shown with the dotted line.

The $z_{\mathrm{c}} \sim S$ relationship with the fixed $\alpha$ parameter and fixed normalization of the integral:

$$
\int f_{S}(z) \mathrm{d} \log z=1
$$

eliminates formally any free parameters in the fitting the analytic function to the nine histograms in Fig. 1:

$f_{S}(z)=\frac{\ln 10}{\Gamma(\alpha) z_{\mathrm{c}}^{\alpha}} z^{\alpha} \mathrm{e}^{-z / z_{\mathrm{c}}}$,

where $\alpha=1.934, \Gamma(\alpha)=0.9739$ is the gamma function and $z_{\mathrm{c}}$ is specified for each sample by the $z_{\mathrm{c}} \sim S$ relationship. Analytic distributions defined in Eq. (11) are shown in Fig. 1 with the solid curves. In all the histograms the model distribution is astonishingly close to the corresponding best three-parameter fit represented by the dotted curves. Most deviations visible in some plots are easily explained by the statistical nature of the problem and/or the large scale structures present in the catalogs based on the localized sky area (Mullis et al. 2001; Barger et al. 2002; Gilli et al. 2003). Systematic shifts between the fits are present in three histograms below $S=10^{-14} \mathrm{cgs}$. It is a direct result of the assumption of a single $z_{\mathrm{c}}=0.59$ value for all three Chandra samples. It is noticeable that the constant width (in $\log z$ ) model fits adequately describe the data over the full range of fluxes. Assuming that the width of the distributions exhibits a genuine dependence on flux, one might expect a monotonic relationship rather than deviations of both signs. In terms of the present considerations, this dependence would produce systematic differences between the three parameter fits and our final model. The actual small differences visible in the NEP, RIXOS and XMS data, apparently do not represent such systematic effects. In the NEP and RIXOS histograms the final model is slightly narrower than the individual fits, while in the XMS sample it is wider.

To effectively use the Eq. (1) one needs the representation of the source counts $\mathrm{d} N(S) / \mathrm{d} S$ over the whole range of fluxes $S$. The parametrization by Moretti et al. (2003) adequately suits the present calculations. The functional form for $N(S)$ proposed by Moretti et al. is a smoothed double power law which accurately reproduces the observed counts below $10^{-11} \mathrm{cgs}$ down to Chandra threshold of $\sim 2 \times 10^{-17} \mathrm{cgs}$. Sources within these flux limits generate more than $90 \%$ of the XRB and smooth extrapolation of the Moretti et al. (2003) counts down to $\sim 3 \times 10^{-18} \mathrm{cgs}$ is consistent with the entire XRB. Substituting all the components into Eq. (1) we finally get the redshift distribution of the XRB photons. It is shown with the solid curve in the bottom right panel in Fig. 1. The same normalization has been applied to facilitate comparison with the distributions derived for the individual samples. Points in the plot are discussed below in the Sect. 7.

A suitable representation of the $b(z)$ distribution has been found using a smooth function of the same form as for the individual redshift histograms. The function:

$b_{\text {fit }}(z)=5.24 z^{1.52} \mathrm{e}^{-z / 0.63}$

reproduces the derived distribution of $b(z)$ with the relative error of less than $4 \%$ for $0.06<z<6$. It is shown with dots in the bottom right panel (with normalization rescaled to conform to all the plots in Fig. 1). 


\section{XRB and supervoids}

The distribution of the XRB photons $b(z)$ peaks at redshift $z \approx 1$ and $50 \%$ of the background originates between the redshifts of 0.4 and 1.4 (for $80 \%$ the redshift limits are 0.2 and 2.1). Thus, very large structures of the matter distribution at redshift within these limits would generate fluctuations of the integral XRB. As an example I discuss below the X-ray signature of the huge void postulated by Rudnick et al. (2007). The arguments based on the radio survey in favor of the void with a radius of $\sim 140 \mathrm{Mpc}$ comoving in Eridanus have been questioned (Smith \& Huterer 2008). Nevertheless, the Integrated Sachs-Wolfe effect operating on extremely large structures of matter remains a valid explanation of the strongest CMB fluctuations.

The redshift separation, $\Delta z$, corresponding to the far side and the near side of the void with the comoving radius $R_{0}$ centered at redshift $z$ is equal to (e.g. Hogg 1999):

$\Delta z \approx \frac{2 R_{0}}{c / H_{0}} \sqrt{\Omega_{\mathrm{m}}(1+z)^{3}+\Omega_{\Lambda}}$

(with the implicit assumption that the diameter of the void is small compared to the void distance). Thus, for example the void of $280 \mathrm{Mpc}$ diameter centered at redshift 0.5 extends between redshifts 0.457 and 0.543 . Using the $b(z)$ distribution we assess the fractional deficit of the XRB $\delta=\Delta b / b$ created by the completely empty region of size $280 \mathrm{Mpc}$. Such a void would generate $|\delta|=5.7 \%$ and $\delta=4.9 \%$ at redshifts $z=0.5$ and 1, respectively. Assuming spherical shape of the void, its angular diameter would be 8.4 and 4.7 at these redshifts. The XRB depression produced by the void should be compared to the XRB intrinsic fluctuations resulting from the discrete nature of sources generating the background. Assuming purely random distribution of sources, the rms fluctuations of the XRB are defined by the source counts $N(S)$ :

$\sigma_{b}=\left[\int_{S_{\min }}^{S_{\max }} \mathrm{d} S S^{2} \omega\left|\frac{\mathrm{d} N(S)}{\mathrm{d} S}\right|\right]^{1 / 2}$,

where $\omega$ is the solid angle subtended by the investigated area. Using the Moretti et al. (2003) counts and $S_{\max }=1 \times 10^{-11} \mathrm{cgs}$ (the amplitude of the XRB fluctuations is dominated by the contribution of sources at the bright end of counts), we get $\delta b / b=0.035$ and 0.024 for the circular areas of radius $2^{\circ}$ and $3^{\circ}$, respectively. Thus, at $z=0.5$ the signal-to-noise ratio for the void detection amounts to $\sim 2.4$. In the case of $z=1$ the $\mathrm{S} / \mathrm{N}$ drops to just 1.4. To reduce the amplitude of the XRB fluctuations one should remove from the XRB the contribution of bright sources. If the $S_{\max }$ is decreased to $1 \times 10^{-12} \mathrm{cgs}$, the significance of the void signal reaches $3.5 \sigma$ at $z=0.5$ and $2.0 \sigma$ at $z=1$. So, only the low redshift voids would produce the XRB deficits significantly stronger than the statistical fluctuations.

Considering the positive CMB detections of supercluster and supervoid structures identified in the Sloan Digital Sky Survey (Granett et al. 2008), the present results show that analysis of the XRB fluctuations cannot provide comparable constraints for the large scale mass distribution.

\section{Redshift distribution of the XRB and the AGN evolution}

To assess the distribution of the XRB generated just by the AGN, I have repeated all the procedures described in the previous sections using the samples constructed exclusively from the AGN. The AGN sources are easily separated from the clusters and

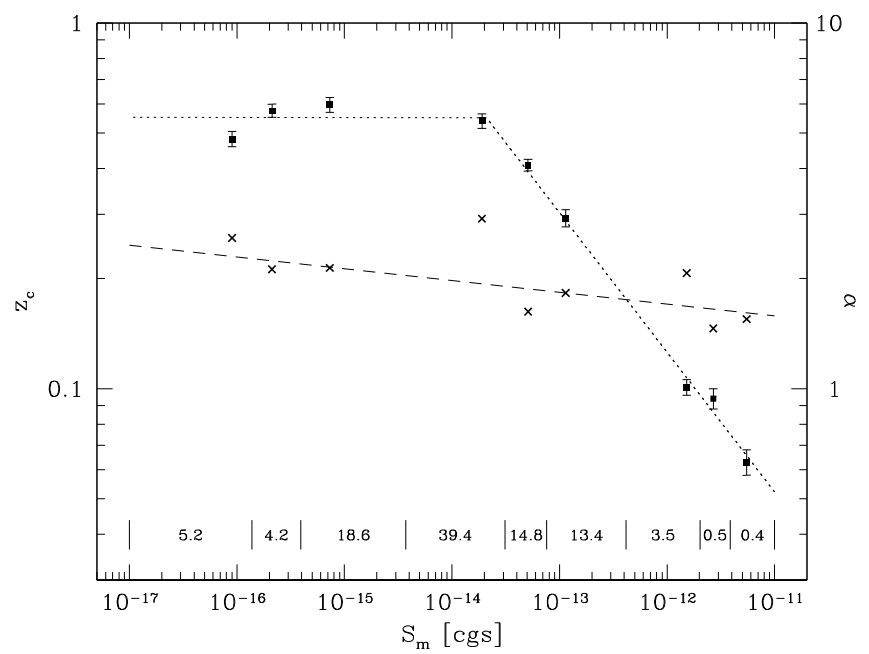

Fig. 3. Distributions of best fitted parameters $\alpha$ and $z_{\mathrm{c}}$ vs. the median flux, $S_{\mathrm{m}}$, in the AGN samples. Points with error bars $-z_{\mathrm{c}}$ (left-hand ordinate), crosses $-\alpha$ (right-hand ordinate). The dashed line represents the regression line for the $\alpha \sim S_{\mathrm{m}}$ relationship; the $z_{\mathrm{c}} \sim S$ function used to model the AGN contribution to the XRB is shown with the dotted lines (see text for details). The vertical bars at the bottom mark the effective flux limits of the analyzed samples; numbers indicate a relative contribution to the XRB generated by the AGN within these limits.

nearby normal galaxies. However, the distinction between the nuclear activity and stellar emitters in the case of distant and weak sources becomes problematic. Such sources are present in both Chandra surveys. One should keep in mind this limitations in the present investigation. Nevertheless, the AGN are a dominating constituent of all the samples exploited in the paper and even the moderate contamination of the AGN subsamples with the off-nuclear sources would not affect significantly our calculations (see below). The numbers in the AGN samples are smaller than in the full samples and parameter estimates are subject to slightly larger uncertainties. Clusters and normal galaxies populate on the average lower redshift bins and the histograms for the AGN analogous to those in Fig. 1 are shifted towards the higher redshifts. We notice also a weak correlation of the best fit $\alpha$ parameter with the source flux - a shape of of the redshift distribution (in $\log z$ bins) varies with $S_{\mathrm{m}}$. In Fig. 3 the values of $\alpha$ in the nine samples are shown with crosses. The regression line of $\log \alpha$ on $\log S$ is used to fix the value of $\alpha$ for the each sample and to calculate the best fit parameter $z_{\mathrm{c}}$. These new $z_{\mathrm{c}}$ are shown in Fig. 3 with the squares. Finally, using the least square method the best fit line

$\log z_{\mathrm{c}}=-(0.382 \pm 0.013) \log S_{\mathrm{m}}-(5.48 \pm 0.17)$

is calculated for six brighter samples to obtain $z_{\mathrm{c}}$ for $S>1.9 \times$ $10^{-14}$ cgs. A constant $z_{\mathrm{c}}$ is assumed for lower fluxes, and the complete $z_{\mathrm{c}} \sim S_{\mathrm{m}}$ relationship adopted for further computations is shown in Fig. 3 with the dotted lines.

The X-ray source counts used in the present case should be limited to the AGN only. Two other major classes of sources contributing to the counts are associated with clusters and nor$\mathrm{mal} /$ starburst galaxies. The analytic formula obtained by Moretti et al. (2003) quite accurately represents counts of all the types of extragalactic sources, but the relative contribution of the each class in the total counts is not well established. One should notice, however, that most of the XRB is produced by sources in 
the middle range of fluxes considered here, while the cluster contribution is significant only at the bright end of counts and the normal and starburst galaxies populate mostly the faint end of counts. Vertical bars at the bottom of Fig. 3 divide the flux range of $10^{-17}-10^{-11} \mathrm{cgs}$ into 9 contiguous bands corresponding approximately to fluxes surveyed by the source samples defined in the paper. The numbers between the bars give the relative contribution of each flux band to the total XRB.

To extract the cluster and starburst galaxies contributions we corrected the total counts in the following way. In the RBS(b) sample clusters constitute $40 \%$ of all the extragalactic sources. The slope of the cluster counts at the bright end amounts approximately to 1.3 (De Grandi et al. 1999). Substantially flatter slope than that for the total counts reduces the relative cluster contribution at lower fluxes. Although the cluster counts are not well constrained below $\sim 10^{-12} \mathrm{cgs}$, their contribution to the total counts drops at the faint end of counts to a negligible level. The source counts attributed to the AGN are assessed by subtracting the cluster counts from the total counts defined by the Moretti et al. (2003) formula. The normal and starburst galaxies are relatively abundant in the CDFN(f) sample. Of 241 extragalactic sources, 147 have been classified as "AGN", 51 as "starburst" and for the other 43 the redshift is unknown. The absolute maximum content of the non-AGN sources in the CDFN(f) sample amounts to $(51+43) / 241 \equiv 39 \%$, assuming that all sources with undetermined redshift are starburst galaxies. The amount of the non-AGN sources at higher flux levels drops quickly. In the XMS sample none extragalactic source with known redshift has been classified as normal or starburst galaxy. The maximum possible contribution of the starburst galaxies at the low flux levels has been accounted for by flattening the slope of the Moretti et al. (2003) counts below $10^{-14} \mathrm{cgs}$ to reproduce the reduction of the AGN in the CDFN(f) sample by $39 \%$. The counts modified this way have been substituted into Eq. (1) to obtain the redshift distribution, $b_{\mathrm{AGN}}(z)$.

One can use the observed distribution of the background flux produced by the AGN to calculate the cosmic history of the luminosity density generated by these objects. The $b_{\mathrm{AGN}}(z)$ distribution is inserted into Eq. (7) which relates the cosmological evolution of the X-ray luminosity density, $\varepsilon(z)$, to the redshift distribution of the background, $b(z)$. Variations of the luminosity density obtained this way are shown in Fig. 4 with the solid curves. The data are displayed in three panels as a function of redshift, $z$, logarithm of $(1+z)$ and the cosmic time, assuming $t_{0}=13.47 \times 10^{9}$ years for the present age of the Universe ${ }^{2}$. One should note, that the plots are based on the soft X-ray emission only and do not include radiation absorbed and re-emitted in different energies.

The accuracy of the present $\varepsilon(z)$ estimate depends strongly on a quality of our $b(z)$ fits. Relatively small numbers of sources at redshifts below $\sim 0.03$ and above $\sim 3$ generate large statistical fluctuations and weakly constrain the analytic fits $b(z)$ in these redshift ranges. Hence, the present estimates of $\varepsilon(z)$ are also subject to large uncertainties at very low and high redshifts. Comparison of our results with some other works is presented in the next section.

In order to assess the importance of the $N(S)$ uncertainties on the present estimates of $\varepsilon(z)$, I have plotted in Fig. 4 with the dotted curves the $\varepsilon(z)$ function using the original Moretti et al. (2003) formula, i.e. assuming no corrections for clusters and starburst galaxies. The discrepancies between both solutions do

\footnotetext{
${ }^{2}$ For the cosmological model defined in Sect. 1 and using the formulae given by Hogg (1999).
}
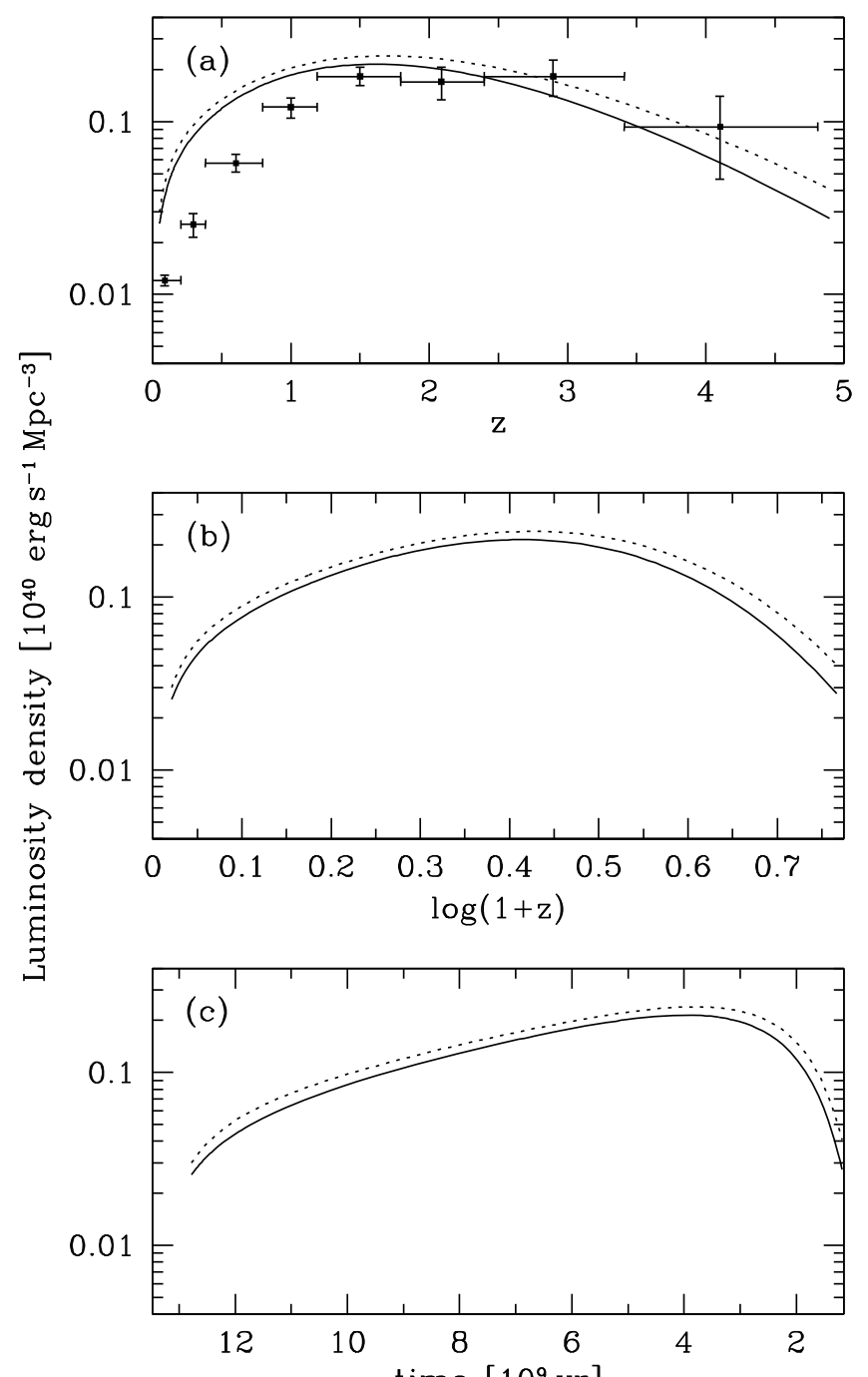

Fig. 4. The cosmic evolution of the X-ray luminosity density generated by AGN as a function of: a) redshift $z, \mathbf{b}) \log (1+z)$ and c) the cosmic time. Solid curve - based on AGN counts; dotted curve - based on total counts (see the text for details); points with the error bars in the panel a) are taken from Hasinger et al. (2005) and represents only the type-1 AGN.

not exceed $20 \%$ for redshifts below $\sim 3$. It implies that our procedure to isolate the contribution of AGN from the total counts, albeit crude, does not contribute significantly to the final errors of $\varepsilon(z)$.

\section{Discussion}

The main objectives of the present investigation, viz. estimates of the redshift distribution of the XRB photons, $b(z)$, and the evolution of the AGN luminosity density, $\varepsilon(z)$, have been achieved using the smooth, analytic fits to the observed source redshift histograms. The present method is conceptionally simple and computationally straightforward. Unfortunately, it does not provide error estimates. The major sources of uncertainties have been indicated in the previous section. Here a quantitative estimate of the errors is discussed.

The errors of the present measurement of $b(z)$ are generated by the statistical nature of the investigated material and a chain of approximations applied to substitute the observed 
redshift distributions centered on a selected fluxes by an analytic function $f_{S}(z)$ continuous in both parameters, $z$ and $S$. In fact, the visual inspection of the analytic fits displayed with the solid curves in Fig. 1 reveals some deviations from the redshift histograms. To estimate the significance of these differences, the calculations have been performed using the actual histograms shown in Fig. 1 with broken solid lines instead of $f_{S}(z)$. For the each value of flux $S$ in the range $10^{-17}-10^{-11} \mathrm{cgs}$, the corresponding redshift distribution has been obtained by the linear interpolation between two histograms from the samples centered on the median fluxes nearest to $S$. The results of this procedure are shown in the lower right panel of Fig. 1 with dots. Generally good agreement between the distribution of points and the solid curve proves that the analytic approximations do not introduce perceptible systematic errors in the present investigation. It appears that the relatively large deviations for three data points (centered at redshifts: $0.087,0.65$, and 1.16 ) result purely from the large scale structures. This is particularly likely for the first bin $(0.075<z<0.1)$, where the discrepancy between the fits is produced entirely by the excess of sources in the localized NEP survey (Mullis et al. 2001).

One should also notice, that the uncertainties of our main results are only weakly affected by the limited statistics of the individual samples and histograms. This is because the final distributions are obtained by averaging the individual distributions and this procedure effectively reduces statistical fluctuations.

In the upper panel of Fig. 4 the AGN emissivity calculated by Hasinger et al. (2005) is shown. The points with the error bars are redrawn here from their original paper. Hasinger et al. (2005) apply more stringent criteria to select sources and use only well defined samples of type-1 AGN. Optically these objects are identified by the broad Balmer emission lines, while using the X-ray criteria, they have unabsorbed spectra indicating low intrinsic column densities. In the present analysis I have included all objects in which the X-ray emission originates in the active nuclei. Thus, our results cannot be compared directly to those by Hasinger et al. (2005). Nevertheless, despite entirely different method applied in the present paper, the distributions show good agreement at redshifts above $\sim 1$. Although most of the apparent discrepancies, which reach a factor of 3 at $z \approx 0.5$, are probably due to the distinct selection criteria of both investigations, one cannot exclude that some differences are caused by unrecognized systematic effects inherent in one or both methods.
The present method of the luminosity density calculations has also some disadvantages. In our approach the absolute luminosities of the individual objects are not determined. Consequently, only the integral luminosity density is obtained, and the cosmic evolution of any selected AGN luminosity class has to be studied by means of the standard methods.

Acknowledgements. This work has been partially supported by the Polish MNiSW grant N N203 395934.

\section{References}

Alexander, D. M., Bauer, F. E., Brandt, W. N., et al. 2003, AJ, 126, 539 Barcons, X., Carrera, F. J., Ceballos, M. T., et al. 2007, A\&A, 476, 1191 Barger, A. J., Cowie, L. L., Brandt, W. N., et al. 2002, AJ, 124, 1839 Barger, A. J., Cowie, L. L., Capak, P., et al. 2003, AJ, 126, 632 Brandt, W. N., \& Hasinger, G. 2005, ARA\&A, 43, 827

De Grandi, S., Böhringer, H., Guzzo, L., et al. 1999, ApJ, 514, 148 Donley, J. L., Rieke, G. H., Pérez-González, P. G., Rigby, J. R., \& Alonso-Herrero, A. 2007, ApJ, 660, 167

Georgakakis, A., Rowan-Robinson, M., Babbedge, T. S. R., \& Georgantopoulos, I. 2007, MNRAS, 377, 203

Giacconi, R., Zirm, A., Wang, J.-X., et al. 2002, ApJS, 139, 369

Gilli, R., Cimatti, A., Daddi, E., et al. 2003, ApJ, 592, 721

Gioia, I., Henry, J., Mullis, C., et al. 2003, ApJS, 149, 29

Granett, B. R., Neyrinck, M. C., \& Szapudi, I. 2008, ApJ, accepted [arXiv: 0805.3695]

Hasinger, G., Miyaji, T., \& Schmidt, M. 2005, A\&A, 441, 417

Henry, J., Mullis, C., Voges, W., et al. 2006, ApJS, 162, 304

Hogg, D. W. 1999 [arXiv: astro-ph/9905116]

Kim, M., Wilkes, B. J., Kim, D.-W., et al. 2007, ApJ, 659, 29

Lehmann, I., Hasinger, G., Schmidt, M., et al. 2001, A\&A, 371, 833

Lehmer, B. D., Brandt, W. N., Hornschemeier, A. E., et al. 2006, AJ, 131, 2394

Mason, K. O., Carrera, F. J., Hasinger, G., et al. 2000, MNRAS, 311, 456

Miyaji, T., Hasinger, G., \& Schmidt, M. 2000, ApJ, 353, 25

Moretti, A., Campana, S., Lazzati, D., \& Tagliaferri, G. 2003, ApJ, 588, 696

Mullis, C. R., Henry, J. P., Gioia, I. M., et al. 2001, ApJ, 553, L115

Ravikumar, C. D., Puech, M., Flores, H., et al. 2007, A\&A, 465, 1099

Reddy, N. A., Steidel, C. C., Erb, D. K., Shapley, A. E., \& Pettini, M. 2006, ApJ, 653, 1004

Rudnick, L., Brown, S., \& Williams, L. R. 2007, ApJ, 671, 40 [Erratum: ApJ, $678,1531(2008)]$

Schmidt, M. 1968, ApJ, 151, 398

Schwope, A. D., Hasinger, G., Lehmann, I., et al. 2000, AN, 321, 1

Silverman, J. D., Green, P. J., Barkhouse, W. A., et al. 2007 [arXiv: 0710. 2461]

Smith, K. M., \& Huterer, D. 2008 [arXiv: 0805.2751]

Stocke, J. T., Morris, S. L., Gioia, I. M., et al. 1991, ApJS, 76, 813

Szokoly, G. P., Bergeron, J., Hasinger, G., et al. 2004, ApJS, 155, 271

Voges, W., Aschenbach, B., Boller, Th., et al. 1999, A\&A, 349, 389

Zheng, W., Mikles, V. J., Mainieri, V., et al. 2004, ApJS, 155, 73 